Hypotheses

\title{
Real-Time Automatic Inspection System for the Classification of PCB Flux Defects
}

\author{
${ }^{1}$ Ang Teoh Ong, ${ }^{2}$ Aouache Mustapha, ${ }^{3}$ Zulkifilie Bin Ibrahim, ${ }^{4}$ Suzaimah Ramli and ${ }^{5}$ Boo Chai Eong \\ ${ }^{I}$ Control Easy Technology Sdn. Bhd, No.4-4, Jalan SP2/2, Serdang Perdana, 43300 Sri Kembangan, Selangor, Malaysia \\ ${ }^{2}$ Department of Electrical, Electronic and Systems Engineering, Faculty of Engineering and Built Environment, \\ Universiti Kebangsaan Malaysia, 43600 Bangi, Selangor, Malaysia \\ ${ }^{3}$ Department of Electrical Engineering, Faculty of Electrical Engineering, Universiti Teknikal Malaysia Melaka (UTeM), Hang \\ Tuah Jaya, 76100, Durian Tunggal, Melaka, Malaysia \\ ${ }^{4}$ Department of Computer Science, Faculty of Defence Science and Technology, Universiti Pertahanan National Malaysia, \\ Kem Sg Besi, 57000 Kuala Lumpur, Malaysia \\ ${ }^{5}$ Technical Development Manager, INTEL, Bayan Lepas, 11900, Penang, Malaysia
}

Article history

Received: 25-06-2015

Revised: 07-07-2015

Accepted: 20-08-2015

Corresponding Author:

Ang Teoh Ong

Control Easy Technology Sdn.

Bhd, No.4-4, Jalan SP2/2, Serdang

Perdana, 43300 Sri Kembangan,

Selangor, Malaysia

Email: sales@controlez.com

\begin{abstract}
Automatic inspection based on a real-time machine vision system may serve as substitute for the manual human visual inspection of flux defects in Printed Circuit Boards (PCBs), which often cause damage on the board in the form of corrosions that harm the assembly. The concept of automatic inspection contributes to the improvement of the manufacturing quality of PCBs and facilitates their approval or rejection. The Automatic Inspection System for Printed Circuit Boards (AIS-PCB) is developed with the capability to identify the defects and the quality of PCBs. It is based on a real time system machine vision. The developed AIS-PCB is capable of detecting, indexing and classifying by measuring the flux defects in PCBs during the re-flow of the real-time process. The AIS-PCB is The total automation control system is the core of the AIS-PCB. This system consist of vision inspection station, mechanical loader and unloader, final decision station and the pneumatic system handler. To detect and classify the quality of PCBs, segmentation in conjunction with Radon transform approaches are used for feature indexing and line detection based on the gradient field of PCB images. The Feed-Forward Back-Propagation (FFBP) model is used to classify the product quality of the PCBs via a learning concept. A number of trainings using the FFBP are performed to learn and match the targets. The images of each PCB classes are used as inputs to the classification module. The obtained results from the classification and rule decision are used to establish the receiver operating characteristic curve. The classifier, which is based on the proposed approach and is tested on the PCBs from a factory's production line, achieves a sorting Coefficient Of Efficiency (COE $>95 \%$ ). The developed AIS-PCB system shows promising results in successfully segmenting and classifying flux defects in PCBs through computerized visual information and facilitates their automatic inspection, thereby aiding humans in conducting rapid inspections.
\end{abstract}

Keywords: PCB Flux Image, Segmentation Approach, Classification Approach, Automatic Machine Vision System

\section{Introduction}

\section{Motivation}

Computer vision techniques are used for the computerized visual inspection of Printed Computer Boards (PCBs), particularly for the assessment of their various defects. The fault detection strategy applies referential inspections methods whereby the manufactured or an artwork board serves as the references without any errors.

Defects of PCB can be divided into two classes: Rejected units referred to as fatal defects and accepted 
units which are of zero defects. Prone to human errors, extensive labor works and unpredictable grading are common while manually inspecting the PCB defects. This attributes makes it disadvantageous to use.

By operating machine vision on PC, inspections can be automated. Major inaccuracies and faults, for example in printing, markings of components, disoriented components, labelling and such can be recognized by the system.

Therefore, automatic inspection based on a real-time machine vision system is recommended as substitute for the manual human visual inspection of PCBs, particularly for assessing their defects and for quality checking to assist humans in conducting rapid inspection.

This essay will concentrate on the PCB flux which is quite challenging to be envisaged by human eye due its fluid and transparent attributes. Possible applications in the manufacturing of PCB shows promises in the suggested approach. By means of artificial intelligence and image processing, fatal defects are easily detectable. Vision inspection station, mechanical loader and unloader, final decision station and the pneumatic system handler are included in the design of the machine which is in fact, a product of the engineering assimilation of electrical, mechanical, software and electronic field.

\section{Literature Review}

In an ordinary PCB gathering process, the assessment of flux imperfections is a key issue in the quality control of PCBs. For precise visual assessment, a framework that is impervious to disparity in light is required and calculations for arrangements are needed. Besides, the arrangement criteria for flux deformities are for the most part founded on human errors and can be altered based on the products.

Complex image acquisition systems that require several inspection algorithms have been investigated and developed to overcome the above mentioned limitations. To date, various PCB assessment calculations have been proposed in the writing.

Tsai and Tsai (2002) proposed a rotation invariant model coordinating patterns in detecting intricate colored images on an element. Based on this study, it occurs that the trouble and computational load for coordinating colored elements in subjective alignments are decreased essentially through $1-\mathrm{D}$ ring-projection coordination. About $6 \mathrm{~s}$ in a Pentium $300 \mathrm{MHz} \mathrm{PC}$ for a subjectively pivoted picture with a size of 256256 pixels and a circular window with a span of 25 pixels is the reckoning time suggested in order to load the color ring projection coordination.

Computation time for images in a fixed orientation using traditional template-matching methods is $20 \mathrm{sec}$.

The first low cost inspection of physical flaws in PCBs is by building up a method for image identification which was explored by Mashohor et al. (2004). This technique utilizes a genetic calculation. After 150 generations, the system effectively recognized a board with physical flaws. Leta et al. (2005) discussed the fundamental issues in automatic inspection and used some PC vision frameworks to gage parts. Parts conformity examination using a non-contact estimation structure has been recognized, particularly for little objects where accurate instruments, for instance, the heading measuring machine, are used. The obtained results are compared with those of conventional measuring instruments. These studies highlight the challenges in automated image inspection in terms of accuracy and the relationship between an image and its corresponding measurement.

Greenberg et al. (2001) also proposed a method of PCB inspection. The proposed method consists of the following steps: Placing multiple PCBs on an inspection panel, defining non identical PCBs by inspecting geometry and features, grouping the said PCBs into at least one cluster based on the amount, location, or orientation of the $\mathrm{PCBs}$, creating a reference image for the said panel defined by a specific cluster and inspecting the said panel by comparing sensed information with the reference image.

Sundaraj (2009) presented the implementation details of the prototype of an automated visual inspection system by utilizing color background subtraction for uneven and absent parts. Amid the drawing processes, the peculiarities that happen on uncovered PCBs were arranged into two primary sorts: Those with absent coppers and those with useless copper. It is noted that open circuit and patterns on PCB occurs during excessive etchings. Meanwhile, the divided etching methodology leaves undesirable conductive materials and structure disfigurements, for instance, extra hole, protrusions, island, small space and short circuit.

The experimental results of the various defective PCBs were also presented.

Mar et al. (2009) displayed a programmed division approach for the discovery of solder joints. Taking into account the luminance quadrature display, the division methodology is anything but difficult to handle and gets great results.

This process requires no special lighting device or setup and uses a simple thresholding and segmentation algorithm. In general, the same techniques should be applied in other types of object segmentation, such as ball grid array, solder paste and IC lead inspection.

Based on color dissemination of solder joints, Zeng et al. (2011) set forward a calculation for allotting and recognizing PCB segments. The proposed methodology investigated the color dispersion of solder joints under three layers of ring LEDs. The proposed strategy viably perceives the vast majority of the segments based on the trial results. 
Image subtraction technique to distinguish PCBs which are exposed to imperfections were utilized in Chauhan and Bhardwaj (2011). This methodology included stacking a reference image which later buffers it for subtraction operation and assesses the PCB lapse. The investigated image was XORed with the reference image. The acquired faulty zones was subjected to particle examination to investigate the deformities as in size, area, percentage and orientation.

A programmed automatic machine vision framework for the investigation of any missing part in PCBs were proposed in Singh and Bharti (2012). The framework principally comprises of two sections:

The learning process where the system is trained on the standard PCB and (b) the inspection process where the $\mathrm{PCB}$ being examined is inspected for any missing component by comparing it with a standard PCB. The proposed system can be deployed in a manufacturing line at a much more affordable price than other commercial inspection systems.

Kaushik and Ashraf (2012) connected a machine vision PCB review framework in the first stride of the assembling process. For the manufacturing of $\mathrm{PCB}$, they initially contrasted the PCB image by utilizing a straightforward subtraction calculation that highlights the primary issue areas with an uncovered PCB. Their center was to identify absconds in PCBs and focus the impact of noise. For this, they inspected the impact of commotion of noise on a PCB picture and at which rate the frequency of noise can be utilized to distinguish the defective image. Among others, additional etchings, short etchings and gaps are the average faults that can be identified.

Bhardwaj (2012) proposed a computerized optical assessment calculation for the PCB examination framework. The proposed method detects holes and defects that occur in the PCB etching process during manufacturing. The object of the PCB inspection algorithm is to develop a more reliable and faster visual inspection system. The inspection process has two stages: The estimation and identification stages. For the measurement stage, single image is measured from the analyzed image and is utilized for identifying deformities. A new graphic investigation framework strategies by using coordinating and RGB channel to supplant the human visual examination of PCB flux deformities (Teoh Ong et al., 2013). This may however, impose threat to the PCB construction in the form of corrosion. But visual investigation framework on the other hand, improves this condition by changing the nature of PCBs, by deciding their adequacy.

This literature review shows that the application of automatic inspection systems in detecting problems in the production and quality of PCBs has not been broadly concentrated on. Be that as it may, this subject constitutes a testing assignment to specialists and further examination is emphatically persuaded by the objectives of mechanical professionals.

\section{Contributions}

The key aim of this work is to provide quantitative data, specifically on the classes of PCBs whose images are collected, to facilitate the implementation of an automated inspection system for the classification of flux defects. Appropriate PCB manufacturing policies, such as quality standards to facilitate approval or rejection of PCBs, are also recommended. However, with a manual human visual system, meeting all these requirements in real time is difficult. An automated inspection system for the cataloguing of PCB flux defects requires accurate information to arrive at good decisions. Thus, a highly effective and robust system is needed. To develop this system, the specific objectives of this study are the following: (1) To extract the PCB images flux as the Region Of Interest (ROI) through flux segmentation approach, (2) to index and extract features from the resulting segmented images and (3) to produce a robust model based on an Artificial Neural Network (ANN) classifier that can distinguish between PCBs with flux defects and those without at a high classification accuracy.

This paper is organized into four sections: Section 1 introduces the context that motivates the work and provides a review of the literature on machine vision inspection in PCB manufacturing. Section 2 discusses the methodology, particularly the tasks involved in the AIS-PCB: Image acquisition and flux segmentation, flux defect classification system and system performance evaluation. Section 3 presents the experimental results and discussion, along with the AIS-PCB design and fabrication. Section 4 concisely provides the conclusion and recommendations of this study.

\section{Research Methodology}

Based on the literature, the detection and classification of defects are the most significant considerations in the configuration and improvement of a programmed PCB assessment framework. Thus, the major tasks involved in developing the AIS-PCB for the classification of flux defects are the following: (1) Image Acquisition and Segmentation (IAS), (2) Indexing Features (IF) and (3) Flux Defect Classification (FDC) combined with examination and evaluation. Several inspection operations are applied in each task, as indicated in Fig. 1, which represents a detailed scheme for the proposed AIS-PCB system in this research.

\section{PCB Image Database}

This work begins with the development of a database from all of the images that represent the flux 
defects of PCBs. All PCBs are positioned uniformly at $0.5 \mathrm{~m}$ from the camera to ensure the efficiency of the training and testing in the FDC stage and to yield improved results. The PCB images are shown in Fig. 2, in which the PCB is classified into two classes: PCB board without flux defects (normal) (Fig. 2a) and with flux defects (abnormal) (Fig. 2b). These flux defects take the form of a shining light in the sagittal view of the PCB images. In addition, a PCB image is considered normal when the flux defects do not appear in the form of shining light as shown in Fig. 2a. All database images are resized to 300 by 300 pixels. In this study, the images must be preprocessed and converted to extract the flux defects as ROI and must then be examined to separate suitable elements to express to each image in a minimized structure. The database contains 100 images, which are all grouped into two classes.

\section{IAS}

\section{Image Acquisition}

The first step in the AIS-PCB procedure is image acquisition and capture. The image captured by the camera contains the image components with or without flux defects to be classified. The digital images of PCB are captured by a camera under controlled environment lighting conditions. The camera is set at a fixed exposure and focus and is then mounted on a machine vision handler at a height of $0.5 \mathrm{~m}$ from the conveyor. The lens camera has a horizontal viewing angle of approximately $90^{\circ}$. The camera is given a Matrox lens; the Matrox camera has a pixel determination of $640 \times 480$ and a picture determination of $7.4 \times 7.4 \mu \mathrm{m}$ per pixel. By means of the Ethernet system interface, the images are sent to the information obtaining framework.

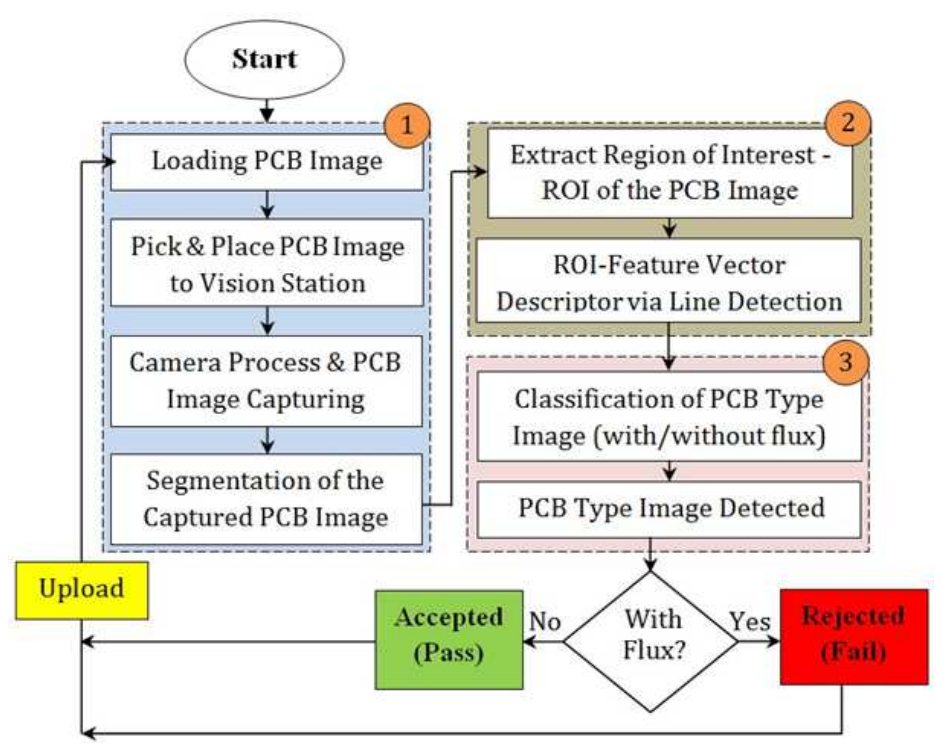

Fig. 1. Scheme for the proposed AIS-PCB system
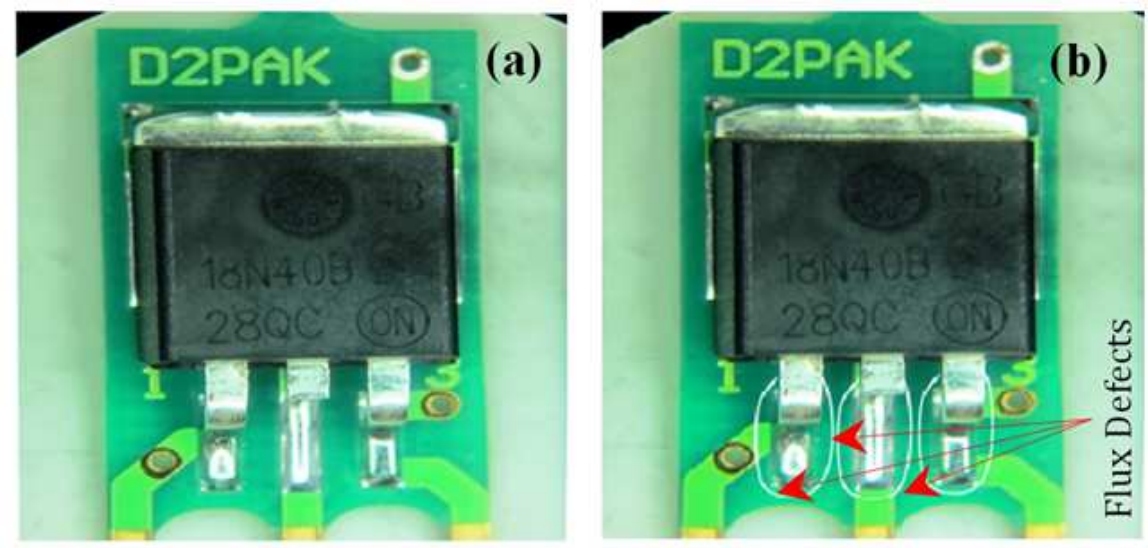

Fig. 2. PCB images: Original image (a) without flux defects (normal) and (b) with flux defects (abnormal) 


\section{Flux Defect Segmentation (FDS)}

The purpose of utilizing the segmentation approach is to detect and extract the PCB image represented with or without defect as ROI. As such, the FDS approach is proposed. The FDS employs different subtractions between PCB image components along with morphological operations to segment the ROI region. The following describes the steps of conducting the FDS:

\section{Input: PCB Image}

\section{Perform First Subtraction (FS):}

- Convert the RGB image into the equivalent HSV image

- Adjust the intensity values of the HSV image by finding the limits of the contrast stretched image

- Obtain the threshold value (L) from the components (V) and (S) using Otsus method

- Perform FS on these two components (V and S) using the following equation: $\mathrm{FS}=\mathrm{V}-(\mathrm{S} \times 5 \mathrm{~L})$

\section{Perform Second Subtraction (SS):}

- Changes the HSV image into a Binary image utilizing the edge value (L) figured in step 1.3.

- Convert the Binary image picture into a Clear image Border (CB) using four-connected neighbors

- $\quad$ Perform SS using the following: SS = FS-(CB-H)

\section{Perform Specific Mask:}

- Obtain the threshold value (L1) from the resulting SS using Otsus method

- Compute mask image by:

$$
(S S<L 1) \Rightarrow \text { mask }=\left\{\begin{array}{c}
{[M N]=\operatorname{size}(\text { mask })} \\
\operatorname{mask}(1: M / 2 ; 1: N)=1 \\
\operatorname{mask}(\text { find }(\text { mask }<0))=0
\end{array}\right.
$$

\section{Perform Morphological Operation:}

- Perform Morphological Operation (MO) on the subsequent mask image with a disk formed shape organizing component with a radius of 11 pixels

- Obtain the substation (L3) between the resulting mask image and $\mathrm{MO}$, given as L3 = mask-MO

- Remove from the L3 every image joined segments that have less than 1000 pixels

- Create a molding the elements with a span of 3 pixels from the subsequent image from step 4.3

- Perform Edge image (ED) on the resulting image from the previous step

- Dilate and erode the ED image

- Obtain the image skeleton from the resulting image from step 4.6

\section{Output: PCB Image with/without Flux Defect}

IF

After obtaining the resulting ROI image through the FDS approach, the next step is to conduct the IF approach to compute and extract the image features. The chosen features must be discriminative and sufficient in describing the PCB image with or without flux defects. The target of the IF methodology is to display a PCB image in its diminished and reduced structure. This encourages and accelerates procedure for the order. To further improve the extraction capability, FDS is performed. In view of Radon Transform (RT), which is employed in this study due to its proficiency, successfully removes the most discriminative and useful elements. As a part of machine learning that involves distinguishing and evacuating the insignificant excess information, feature extraction is normally used. In this study, the component vectors that can extraordinarily display two PCB classes must be resolved so that the elements can be utilized for the organization of PCB images with or without flux imperfections. The following describes the steps of conducting the feature extraction procedure (Hilund, 2007):

Step 1: The first step is to center the PCB images resulting from the FDS approach by subtracting a partial half of the width from every $x$ coordinate and subtracting another half from every y coordinate at its height.

Step 2: The formula of the abstract line is given as $y$. $=a x+b$. As observed in Fig. 3a, the inclination is $a=-\cos (\theta) / \sin (\theta)$ and the intersection with the $y-$ axis is $\mathrm{b}=\sin (\theta)$ which are in accordance with $\rho$ $=\mathrm{x} \cos (\theta)+\mathrm{y} \sin (\theta)$. These parameters are resolved for every mixture of $(\theta)$ and $(\rho)$. The greatest ( $\rho$ max) is situated to be equivalent to the length of the diagonal of the image. Like the directions of $\mathrm{x}$ and $\mathrm{y}$, the $(\rho)$ directions are likewise centred. Figure $3 \mathrm{~b}$ outlines the determination of the most extreme (maximum) and least estimations (minimum) of $\mathrm{x}$ and $\mathrm{y}$.

Step 3: The greatest and least estimations of $\mathrm{x}$ and $\mathrm{y}$ are resolved to decrease the quantity of essential calculation.

Step 4: Whether $\mathrm{x}$ or $\mathrm{y}$ is utilized as the variable, how the base and greatest estimation of the said variable is computed relies upon which of the four regions is delineated, as indicated in Fig. 4. For the angle $(\theta)$, the selection of $\mathrm{x}$ as the variable when the summation line has a flat out slant of more than 1 , which will bring about a few pixels to be skipped. Neither $\theta=0$ nor $\theta=$ 180 is incorporated in light of the fact that the line has infinite inclination.

Step 5: In (Hilund, 2007), computations are based on the directions and the base and greatest estimations 
of the variable relying upon the angle $(\theta)$ is given as in Equation 2 to 5 :

$$
\begin{aligned}
& \left(0^{\circ}<\theta \leq 45^{\circ}\right) \Rightarrow\left\{\begin{array}{l}
y_{\text {max }}=\min (-a \times m+b, n-1) \\
y_{\text {min }}=\max (a \times m+b,-n) \\
\text { for } y \in\left[y_{\text {min }}: y_{\text {max }}\right] \mapsto x=\frac{y-b}{a}
\end{array}\right. \\
& \left(45^{\circ}<\theta \leq 90^{\circ}\right) \Rightarrow\left\{\begin{array}{c}
x_{\text {max }}=\min \left(\frac{-n-b}{a}, m-1\right) \\
x_{\text {min }}=\max \left(\frac{n-b}{a},-m\right) \\
\text { for } x \in\left[x_{\text {min }}: x_{\max }\right] \mapsto y=a \times x+b
\end{array}\right. \\
& \left(90^{\circ}<\theta \leq 135^{\circ}\right) \Rightarrow\left\{\begin{array}{c}
x_{\text {max }}=\min \left(\frac{n-b}{a}, m-1\right) \\
x_{\text {min }}=\max \left(\frac{-n-b}{a},-m\right) \\
\text { for } x \in\left[x_{\text {min }}: x_{\max }\right] \mapsto y=a \times x+b
\end{array}\right.
\end{aligned}
$$

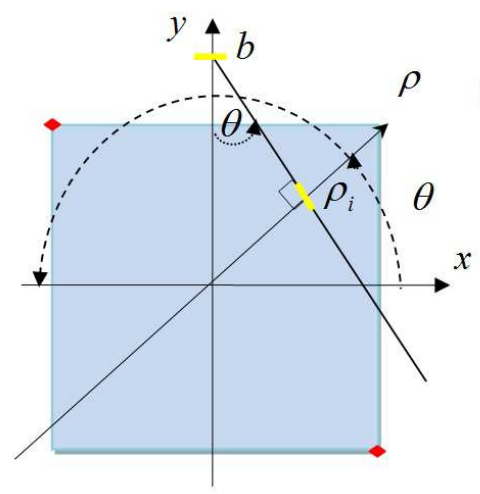

(a)

$$
\left(135^{\circ}<\theta \leq 180^{\circ}\right) \Rightarrow\left\{\begin{array}{c}
y_{\text {max }}=\min (a \times m+b, n-1) \\
y_{\text {min }}=\max (-a \times m+b,-n) \\
\text { for } y \in\left[y_{\text {min }}: y_{\text {max }}\right] \mapsto x=\frac{y-b}{a}
\end{array}\right.
$$

where, $m$ and $n$ are the half-width and the half-height of the fragmented PCB image, correspondingly. $a=-\cos (\theta) / \sin (\theta)$ is the inclination, $b=\rho / \sin (\theta)$ is the crossing point (intersection) with the $y$ axis and_max is the extent of the diagonal of the image and $\rho$ given as Equation 6 and 7:

$$
\begin{aligned}
& \rho_{\max }=\sqrt{2 m^{2}+2 n^{2}} \\
& \text { for } r \subseteq\left[1: \rho_{\max }\right] \Rightarrow \rho=r-\frac{\rho_{\max }}{2}
\end{aligned}
$$

After the projection information are acquired, the normal quality is utilized to construct the component vector. For this situation, the normal is acquired at $180^{\circ}$ pivot of the point $(\theta)$, so that the span of the component vector of the PCB image with or without flux imperfections is exhibited at $180 \times 1$.

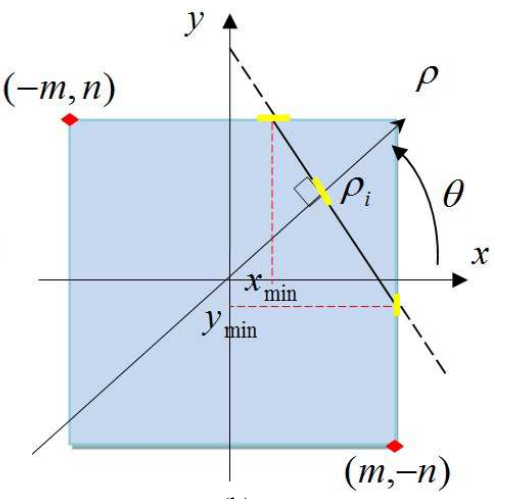

(b)

Fig. 3. Illustration of (a) Steps 1 and 2 and (b) the determination of the maximum and minimum values of $x$ and y in Step 3

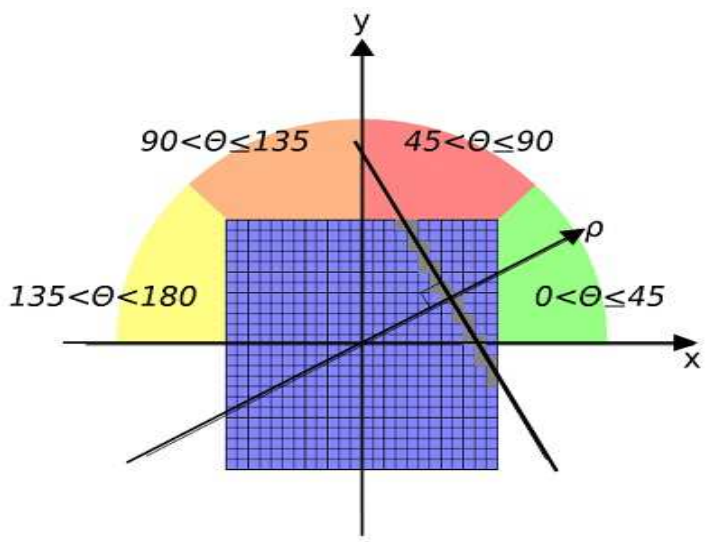

Fig. 4. Selection of angle (step 4) (Hilund, 2007) 


\section{$F D C$}

Propelled by natural frameworks of human, for example, the brain, ANN is a data handling model. It is capable of showing an astonishing number of qualities that is similar of the human cerebrum. The important component of this thought is the innovative structure of the data handling framework or system. It is made out of an expansive number of exceedingly interconnected handling components (neurons) working as one to take care of particular issues. In this way, an ANN can be a powerful tool in various engineering applications (Aouache et al., 2011). Figure 5 shows the schematic of ANN topology. The neurons are associated through a link that connects them together. Every connection has a weight that is duplicated with the transmitted pointer in the system. Every neuron has an actuation capacity to focus on the result. Activation capacities, especially non-linear functions like sigmoid, happen in numerous kinds.

ANN is prepared by experience when an obscure information is connected to the system (Hagan et al., 1996). The purpose of an input layer is to get information from external world as demonstrated in Fig. 5a. The purpose of the output layer is to convey the outcome or forecast the ANN to the outside world. The concealed or hidden layer connects the output layer to the input layer. The role of the hidden layer is to separate and recall helpful elements and sub features from the input examples to anticipate the result of the system network (estimations of the output layer). The output of the neuron net demonstrated in Fig. $5 b$ is acquired utilizing Equation 8:

$$
\begin{aligned}
& y(t-1)=a \sum_{j=1}^{n}\left(w_{i j} x_{i j}(t)-\theta_{i}\right) \text { and } f(\cdot) \\
& =\sum_{j=1}^{m}\left(w_{i j} x_{i j}(t)-\theta_{i}\right)
\end{aligned}
$$

where, $X=\left(X_{1}, X_{2}, X_{3}\right)$ signifies to $\mathrm{m}$ information connected to the neuron, $w_{i j}$ represent the signifies for input $x_{i}, \theta_{i}$ a predisposition quality and $a($.$) is an$ activation function.

The primary distinction among the system sorts lies in the kind of learning calculations and actuation capacity utilized by the hidden neurons. Various calculations may be utilized as a part of preparing ANN models; the greater part of them can be seen as a clear use of streamlining hypothesis and factual estimation. Most of the calculations utilized as a part of preparing ANNs utilize a type gradient descent, which is accomplished by essentially taking the cost function concerning the system parameters and after that changing those parameters into a gradient direction. The most famous of these calculations is the backpropagation algorithm, which has diverse variations. Standard back propagation is a sloped gradient drop calculation. Determining which training algorithm is accurate for a given problem is very difficult and is usually determined through trial and error (Negnevitsky, 2005; Yager and Zadeh, 1994).

\section{Selecting a Topology for a Multilayer Perceptron (MLP) Network}

Each phase of any ANN undertaking some experimentation to set up a suitable and stable system for the task. Experimentation may be reached out from assembling several systems, halting and testing the system at diverse phases of learning and instating the system with distinctive weights. Before choosing the topology of the system, selecting the proper input and output parameters is important. Every system must be tried and examined to focus the most proper system for a specific venture.

Rafiq et al. (2001) the input parameters decides the spatial measurements of the system. The quantity of solutions are determined by the quantity of output in the system. Excess of input and output parameters can definitely halt off the learning procedure. This is because very few arrangements of information may give inadequate data about localized features and may block the system which will cause poor system reaction to concealed information. The quantity of input and output parameters is ought to be augmented as soon as possible.

ANNs have numerous sorts of architecture planning; be that as it may, the multilayer feedd forward neural system is the most largely employed (Rafiq, 2001; Aouache et al., 2011; Hussain et al., 2001).

Multilayer feed forward neural system regularly comes with a data layer consisting an input layer and several hidden layers. In these systems, neurons are masterminded in layers and neurons in distinctive layers, which will be connected to each other. The input data are connected to the input layer while the output adds to the input signal. Hidden layers are basically input and output signals which are in between. The input signs are proliferated in a progressively changed manner before they extent to the output layer.

In this research, the back-propagation learning calculation is led in a feed forward, single concealed layer neural system. The Scaled Conjugate Gradient (SCG), Levenberg Marquardt (LM), adaptive learning rate (GDX) algorithm, sloped gradient descent and are the calculations utilized as a part of this study. Output and hidden layers both uses the Hardlim exchange function. In the preparation, numerous quantities of neurons $(6,8,10$ and 12) are connected to hidden layer to precisely calculate the output. 

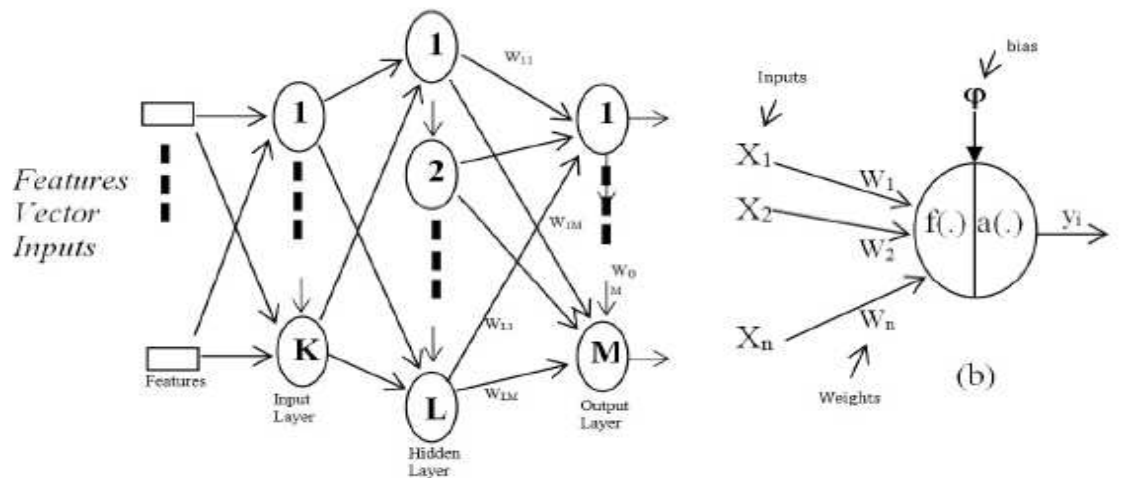

(a)

Fig. 5. Schematic of an ANN topology (Aouache et al., 2011)

Table 1. Statistical criteria for the evaluation of the models in the training stage

\begin{tabular}{llr}
\hline Linguistic terms & Statistical criteria & Calculation formula \\
\hline Root mean squared error & Symbol & $\sqrt{\frac{1}{n} \sum_{i=1}^{n}\left(y_{i}-\hat{y}_{i}\right)}$ \\
Mean absolute error & RMSE & $\frac{1}{n} \sum_{i=1}^{n}\left|y_{i}-\hat{y}_{i}\right|$ \\
Coefficient of efficiency & MAE & $\sum_{i=1}^{n}\left(y_{i}-\hat{y}_{i}\right)$ \\
& COE & $1-\frac{i=n}{i+n}\left(y_{i}-\hat{y}_{i}\right)$
\end{tabular}

\section{MLP Performance Evaluation}

The performance of the classifier is measured using statistical criteria and the Receiver Operating Characteristic (ROC) curve.

\section{Statistical Criteria}

The statistical criteria for the evaluation of the models in the training stage are given in Table 1. For each parameter, the estimated values denoted by $\hat{y}_{i}$, are compared with the measured values denoted by $y_{i}$. The methods for the statistical analysis include the Mean Absolute Error, Coefficient of Efficiency and Root Mean Square Error.

\section{$R O C$}

Brown and Davis (2006) the results identified is linked with the estimation of the sensitivity and specificity of the ROC curve set up. Meanwhile the second execution results are viewed as the measurement for the Area Under the Curve (AUC). The ROC curve is a factual measure that studies the execution of an envisioned or diagnostic framework, especially its capacity to be contingent and precisely identify variation from the norm (true positive) without conveying false identifications. As such, ROC investigation offers an efficient examination of the sensitivity and specificity of a conclusion.

Sovierzoski (2008) and Tilbury (2000), describes the accuracy of The True Positive (TP) and True Negative (TN) is based on the grouping of a talented human utilizing the classifier. On the contrary, the False Positive (FP) and False Negative (FN) files portray an error in this arrangement. At the point when all attestation examples are introduced to the classifier, the measurable indexes are calculated, including sensitivity and specificity. Sensitivity $S_{n}$ is the ability of the classifier to recognize the positive example from the really positive examples. Its value arrays from 0 and 1. Smaller FN uncovers a higher sensitivity of the test. The abscissa axis is used to set the sensitivity values as in Equation 9:

$$
S_{n}(e, t)=\frac{\Sigma T P(e, t)}{\Sigma T P(e, t)+\Sigma F N(e, t)}
$$

In Equation 10 the specificity $S_{p}$ is designed along the axis to display the capacity of the ANN classifier to distinguish the negative pattern from the genuinely negative examples. $S_{p}$ shifts between $[0,1]$, where a lesser value numbers of $\mathrm{FN}$ is an indication of higher specificity of the test: 


$$
S_{p}(e, t)=\frac{\Sigma T N(e, t)}{\Sigma T N(e, t)+\Sigma F P(e, t)}
$$

\section{$A U C$}

According to Purves (1992) the AUC can also be used as an extra measure to break down the viability of the classifier under ROC curve. The least complex procedure is to figure the AUC inside the ROC curve, which is a part of the unit square. Subsequently, the estimation of AUC regularly satisfies these disparities: 0 $<$ AUC $<=1$. The AUC is computed under the ROC curve by summing the regions of the trapezoids. The trapezoidal tenet is applied as a part of this study to get the AUC. In the event that the area is cut into vertical sections, every fragment will form a trapezoid. The general AUC is controlled by including these fragments together. An AUC around 1 signifies a great degree of analytic test (Bradley, 1997):

$$
A U C=\frac{1}{m n} \sum_{i=1}^{m} \sum_{j=1}^{n} 1_{p_{i}>p_{j}}
$$

With $i$ runs running over all $\mathrm{m}$ information with true label 1 (anticipated effectively); $j$ runs over all $n$ information points with true label 0 (anticipated mistakenly) and $p_{i}$ and $p_{j}$ signifies the likelihood score allocated by the ANN classifier as for the points $i$ and $j$, individually.

\section{Results and Discussion}

\section{Segmentation Performance}

The performance of the PCB original images can be enhanced by optimizing the parameters of the proposed segmentation algorithm. Figure 6 shows the results of the FDS using PCB images.

\section{IF}

The images of the $\mathrm{PCB}$ representing the IF approach to analyzing two different PCB images with and without flux defects appear in Fig. 7. The figure shows the image of the PCB in both classes, the final segmentation images, Radon scaled data and Radon feature vectors as final output of each image processed using the IF approach.

\section{ANN Model Results}

This segment talks about the assessment of the execution of the ANN models in the PCB unique images. The execution of the ANN models can be improved by enhancing the parameters relying upon the quantity of nodes in the hidden layer and the learning calculations. The learning calculations are analyzed by changing the quantity of hidden nodes used to prepare the ANN models 6 to 12. Each ANN model is taking into account the same PCB images yet diverse learning algorithms.

\section{Training Stage}

ANN topologies with different quantities of hidden layer neurons are then prepared by means of weighting the statistical preprocessed inputs. The related test outcomes (RMSE, MAE and COE) are shown in Table 2.

Table 2 demonstrates that the precision is enhanced by diminishing the quantity of hidden neurons as showed by the smaller RMSE and by the MAE and COE qualities drawing closer to 1 . Past a specific number of hidden layers, the acquired lapses starts to increment, together with the complexity of the ANN. Moreover, the meeting of target error rate (1e-007) takes more cycle resulting in a prolonged delay. In light of the statistical measures for the ANN models displayed in Table 2, the LM calculation shows promising results in respect with SCG and GDX calculations. Among the LM calculations, the LM-[15 10 1 1 calculation shows the most ideal topology. This topology brings about a mean RMSE value of 0.00185 , mean MAE value of 0.017391 and mean COE value of 0.99963 .

The capacity of ANN-MLP to model the IF in the training stage for the PCB image using the proposed approach is examined. Figure 8 illustrates plots of the actual ANN-MLP output (in red), target output (in blue) versus the normalized IF, (a) prior to training and (b) after training the PCB image using LM-[ll 15 10 1]. This three-layer architecture using the hardlim transfer function is determined as the best neural architecture. Using the best ANN-MLP structure, several trainings in classifying the PCB flux images are conducted. The best performance during training is achieved with the lowest RMSE, MAE and the highest efficiency of COE.

\section{Testing Stage}

In the testing stage, results are generated and analyzed using IF. A ROC curve from the testing phase of the ANN framework is additionally determined. It measures how well a decision system positions variables (data points) to diminish the likelihood of being dynamic and active (positive class). The analysis positions the elements that create the AUC score forecasts of the testing data accuracy. To evaluate the execution of the characterization of the PCB images, the whole dataset is analyzed in the testing stage.

Figure 9 shows the ROC graphs of the PCB image with or without flux classification performed by the ANN based on the best algorithm, LM-[15 1010 1]. 


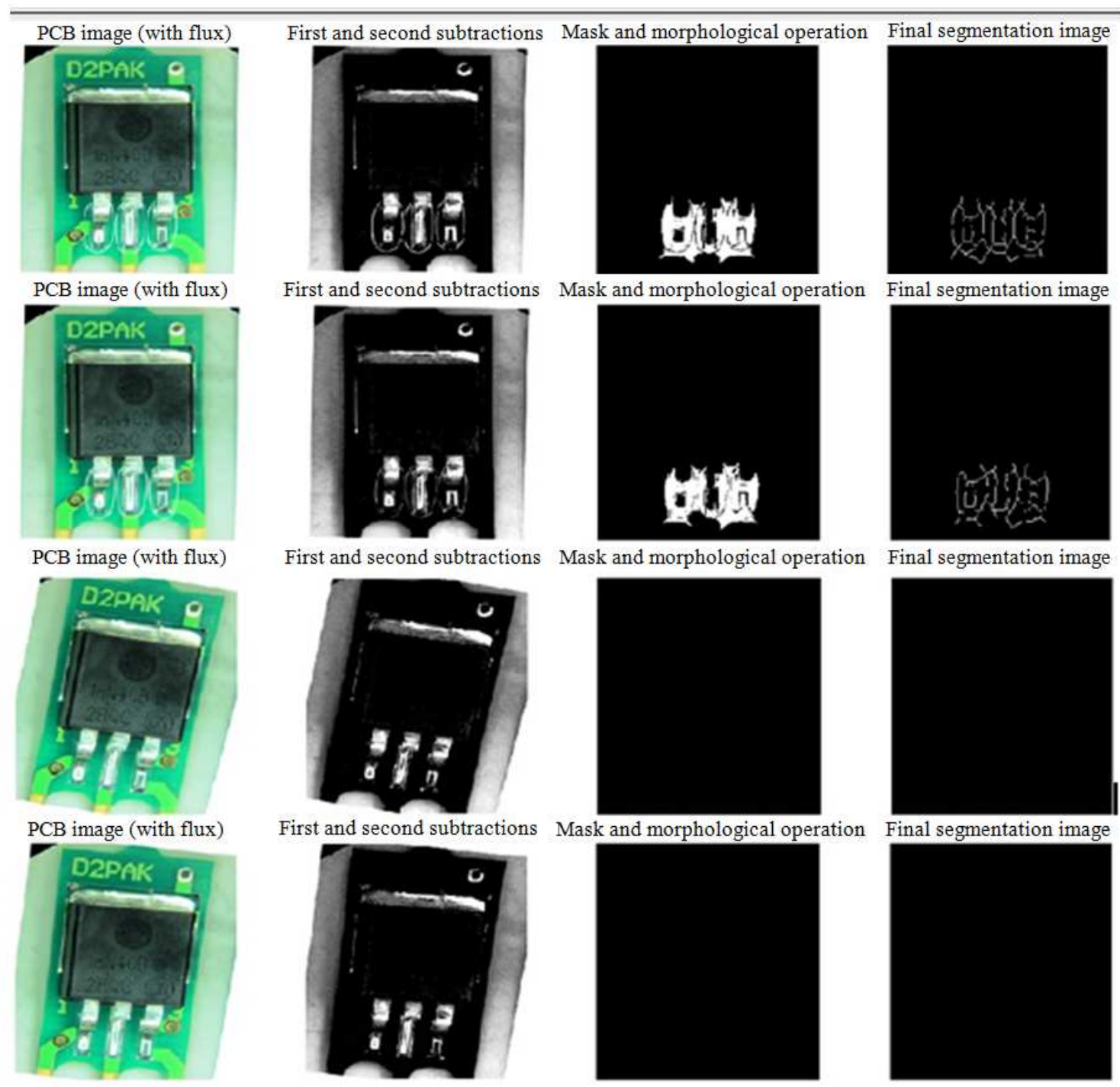

Fig. 6. Results of the FDS process

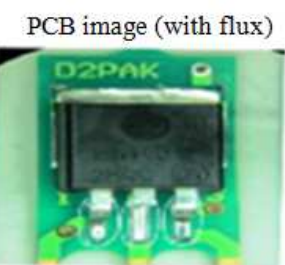

PCB image (with flux)

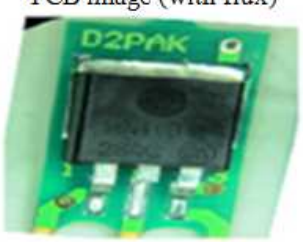

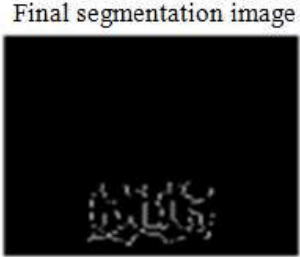

Final segmentation image
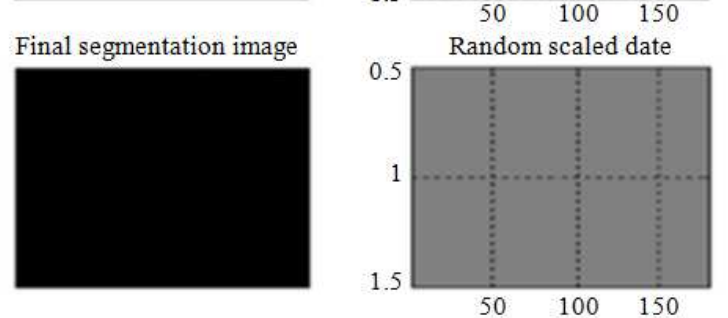
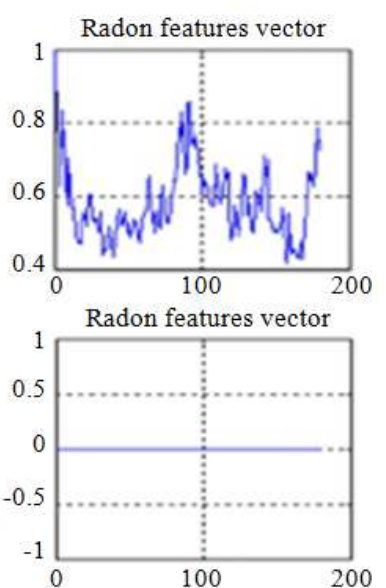

Fig. 7. Results of the IF process 


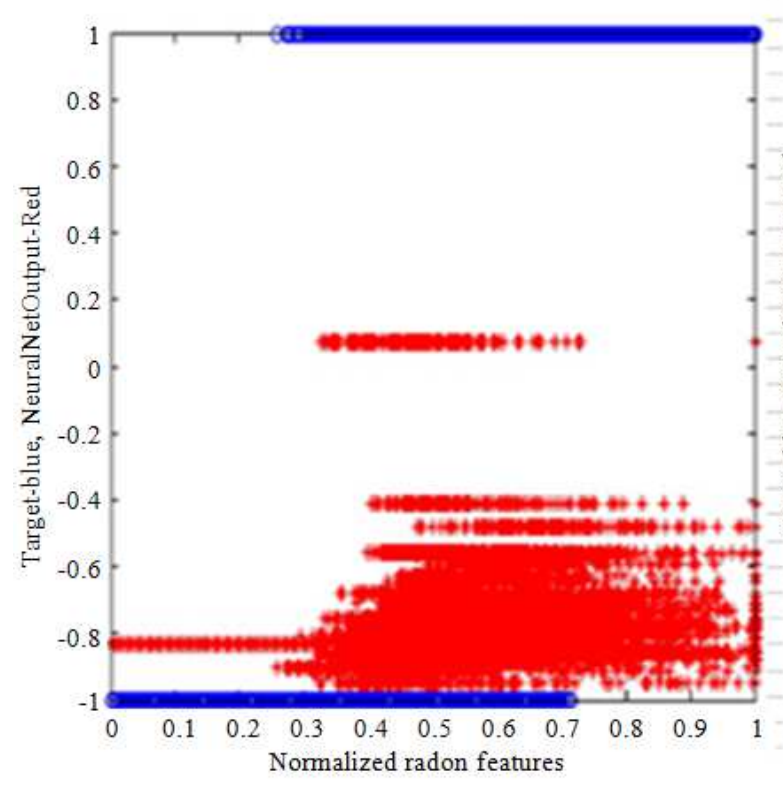

(a)

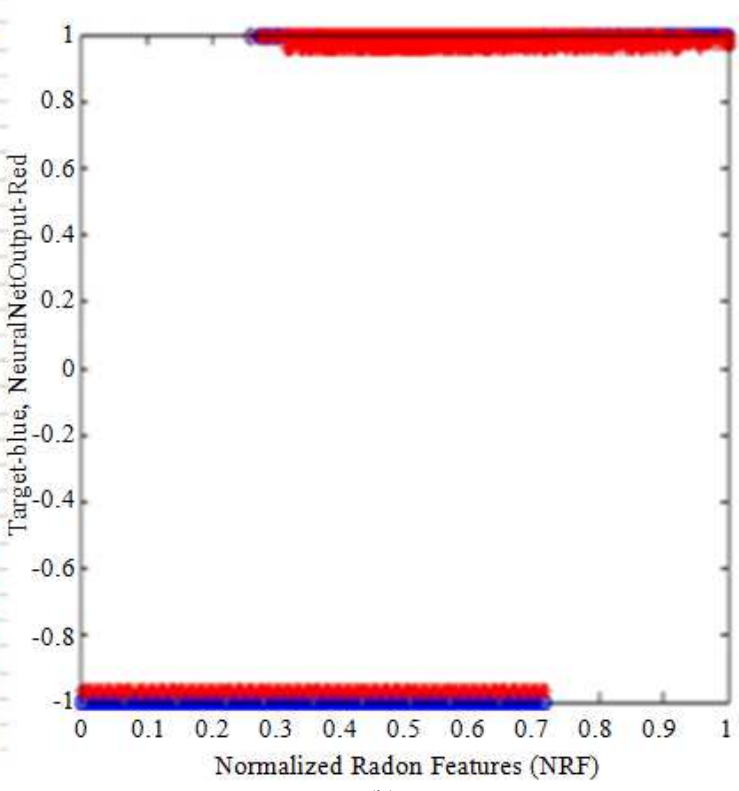

(b)

Fig. 8. Classification performances of ANN (a) prior to training and (b) after training
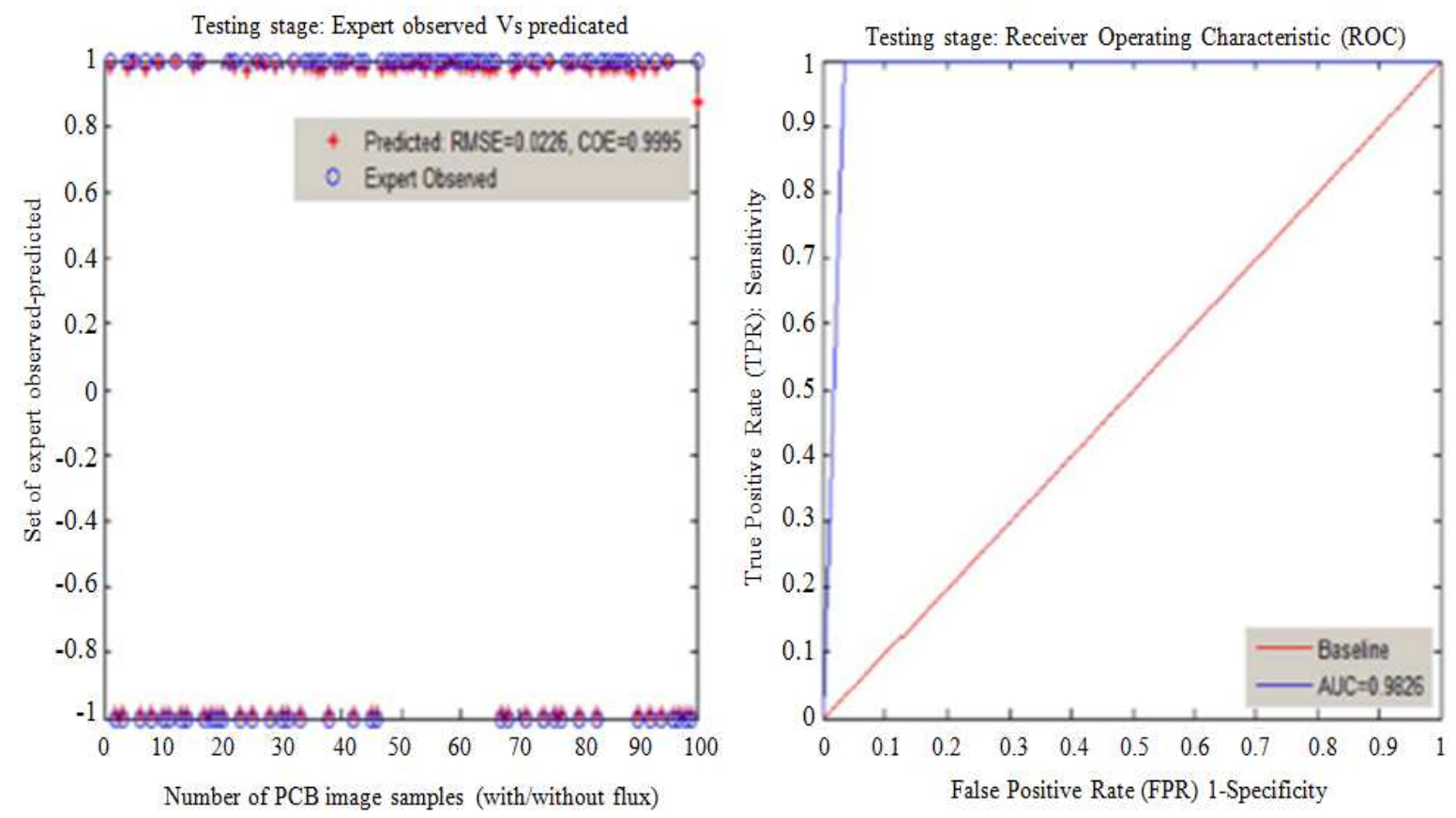

Fig. 9. Classification performance of ANN in the testing stage

Figure 9 demonstrates the execution results accomplished by the ANN decision system by utilizing the best topology accomplished as a part of the preparation stage. The execution results got from the testing stage are 0.0226 for RMSE, 0.9995 for COE and 0.9826 for AUC score as shown in Fig. 9 (left and right). These results indicate the capability of the ANN with topology LM-[15
10 1] to model the IF with high performance in classifying PCB images with or without flux. Based on the statistical measures for the ANN models derived from the best learning algorithms, which are shown in Table 3, the quantitative measurement of LM-[15 10 1] is shown to have the highest COE and AUC scores and the lowest RMSE among the best learning algorithms. 
Table 2. Statistical measure from ANN-models (training stage) for distinctive learning algorithm recreation in between of measured and inspected

\begin{tabular}{|c|c|c|c|}
\hline \multirow[b]{2}{*}{ Learning algorithms } & \multicolumn{3}{|c|}{ Statistical measure: Training stage } \\
\hline & RMSE & MAE & $\mathrm{COE}$ \\
\hline LM-[15 61 l 1$]$ & 0.019742 & 0.018763 & 0.99958 \\
\hline LM-[ $\left.\begin{array}{llll}15 & 8 & 1\end{array}\right]$ & 0.025624 & 0.023910 & 0.99929 \\
\hline LM-[15 10 1] & 0.018583 & 0.017391 & 0.99963 \\
\hline LM-[ $\left.\begin{array}{lll}15 & 12 & 1\end{array}\right]$ & 0.030167 & 0.027491 & 0.99902 \\
\hline $\mathrm{SG}-\left[\begin{array}{lll}15 & 6 & 1\end{array}\right]$ & 0.025621 & 0.021485 & 0.99929 \\
\hline $\mathrm{SG}-\left[\begin{array}{lll}15 & 8 & 1\end{array}\right]$ & 0.030843 & 0.026931 & 0.99897 \\
\hline $\mathrm{SG}-\left[\begin{array}{lll}15 & 10 & 1\end{array}\right]$ & 0.026838 & 0.024668 & 0.99922 \\
\hline $\mathrm{SG}-\left[\begin{array}{lll}15 & 12 & 1\end{array}\right]$ & 0.024554 & 0.021506 & 0.99935 \\
\hline GDX-[1 $\left.\begin{array}{lll}15 & 6 & 1\end{array}\right]$ & 0.030748 & 0.026793 & 0.99898 \\
\hline 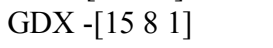 & 0.031373 & 0.028363 & 0.99894 \\
\hline GDX -[15 10 1 1$]$ & 0.031337 & 0.029535 & 0.99894 \\
\hline 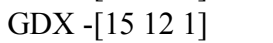 & 0.030965 & 0.026722 & 0.99689 \\
\hline
\end{tabular}

Table 3. Statistical measure form ANN-models (testing stage) for best learning algorithms reproduction between measured and estimated

\begin{tabular}{|c|c|c|c|}
\hline \multirow[b]{2}{*}{ Learning algorithms } & \multicolumn{3}{|c|}{ Statistical measure: Testing stage } \\
\hline & RMSE & $\mathrm{COE}$ & AUC \\
\hline LM-[llllll 15 l & 0.022628 & 0.99945 & 0.9826 \\
\hline $\mathrm{SCG}\left[\begin{array}{lll}15 & 12 & 1\end{array}\right]$ & 0.213760 & 0.95100 & 0.9618 \\
\hline GDX $\left[\begin{array}{lll}15 & 6 & 1\end{array}\right]$ & 0.220580 & 0.94782 & 0.9599 \\
\hline
\end{tabular}

\section{AIS-PCB Configuration System Setup}

\section{AIS-PCB System Overview}

In the experiment, as depicted in Fig. 10, the AIS$\mathrm{PCB}$ is designed and set up to test and implement the classification of PCB images in real time. A computer vision measurement system using digital images can be divided into the following steps: (1) Image acquisition and segmentation, (2) indexing and feature extraction and (3) classification:

- Picture obtaining: An optical framework accumulates a picture, which is then changed over to an advanced configuration and put away into the PC memory

- Image segmentation: In this task, the FDS approach uses different subtractions between PCB image components, along with morphological operations, to segment the flux region

- Feature extraction: RT is used to index and extract features from the resulting segmented images

- Classification and decision: A robust classifier is developed to distinguish PCB classes

\section{AIS-PCB System Design}

The discovery of the PCB ux imperfections are Matrox camera, Matrox frame grabber and Matlab programming, Dell PC, Advantech-DAQ and mechanical automated handler with electro-pneumatic framework are the respective automatic modules of the machine vision framework. The machine vision framework developed with these parts is indicated in Fig. 11:

- Matrox camera specifications: Crystal-clear color images with an effective pixel resolution of $640 \times 480$, pixel size of 7:4×7:4 $\mu \mathrm{m}$ and frame rate of $110 \mathrm{fps}$

- Frame grabber particulars: Standard simple monochrome color PCI frame grabber with two feature decoders, analog composite (CVBS), or Y/C NTSC/PAL input through BNC connectors, flat and/or vertical flip/horizontol, sub sampling to 1/sixteenth of a recorded frame

- PC particulars: Third Generation INTEL CORE i53330 processor 8 GB DDR3 SDRAM, $1600 \mathrm{MHz}-$ 24 GB, 3.51 TB 7200 RPM SATA Hard Drive, $1 \mathrm{X} 1 \mathrm{~TB}$

- DAQ particulars: 48 TTL advanced I/O lines, copies mode 0 of 8255 PPI, buffered circuits for higher driving limit than 8255 , interrupt handling, clock/counter intrude (timer and counter) capacity, supports both dry and wet contact and compatible with USB 2.0 


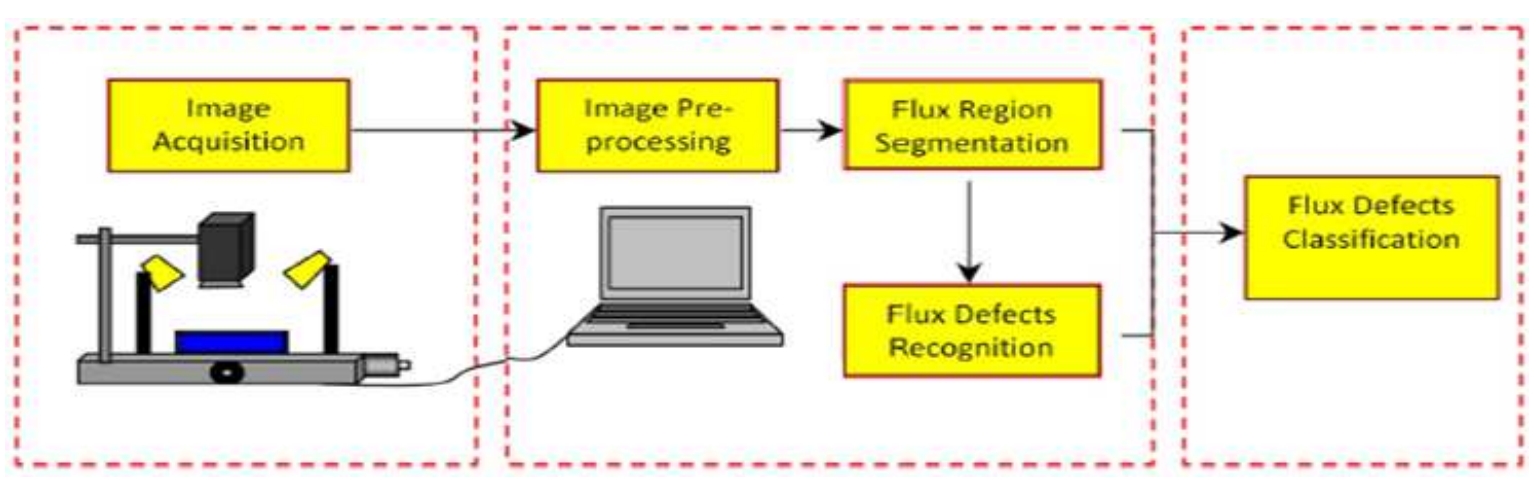

Fig. 10. Overview of the proposed AIS-PCB automatic inspection

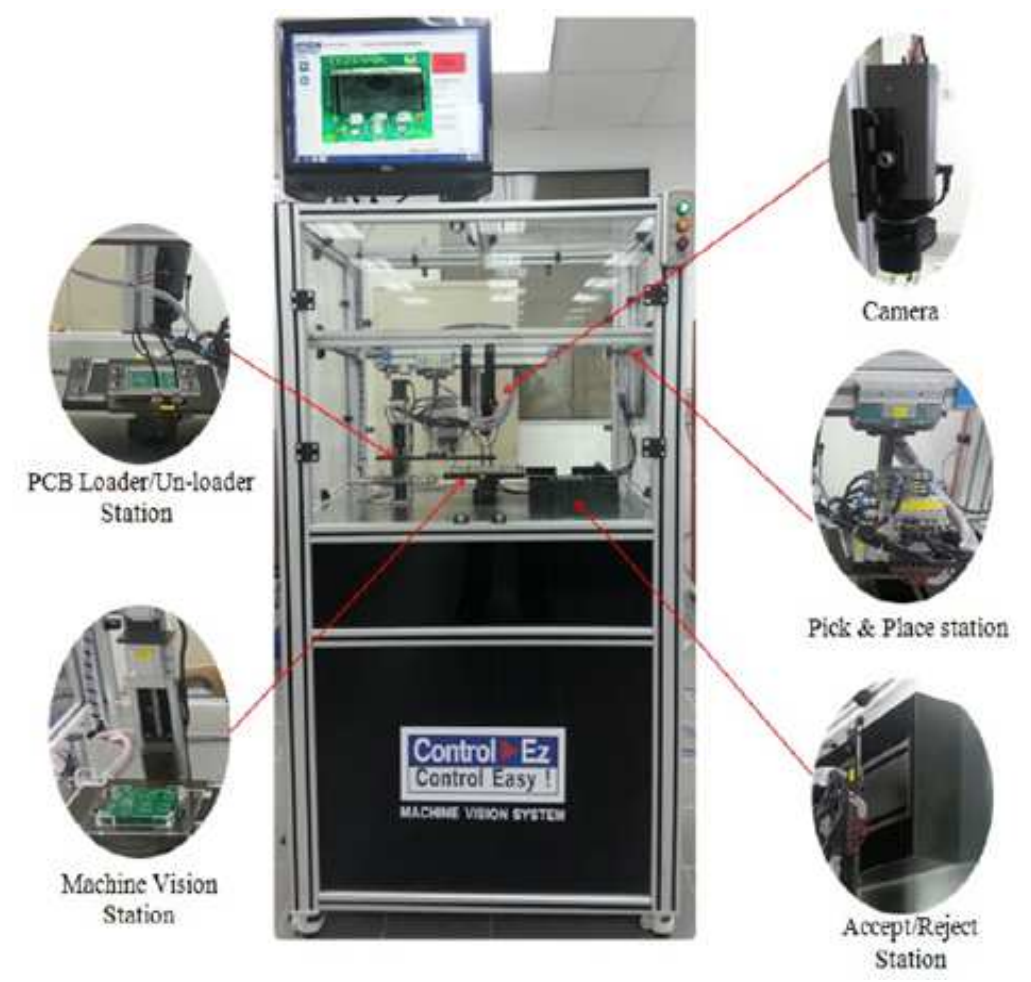

Fig. 11. Design of the real-time AIS-PCB

The AIS-PCB machine vision framework composed and manufactured with the aggregate computerization control system that has a mechanical PCB loader/unloader, pneumatic system handler with vacuum cap, vision investigation automated station and last electro pneumatic arrangement station (acknowledge or reject). A PCB is basically picked from a stacker utilizing a vacuum cap and put on the vision station straightforwardly underneath a camera, which catches a picture of the PCB and sends it to the $\mathrm{PC}$ for inspection. The aftereffects of the assessment are demonstrated on a screen. The inspected PCB is then put at its separate position in light of whether it is acknowledged or rejected.

\section{Conclusion}

This research represents an effort to develop a new automatic inspection system for PCBs. The proposed AIS-PCB is capable of classifying PCB flux defects and therefore of ensuring high PCB manufacturing quality. The segmentation approach provides an index guide for flux defects, which act as powerful tools for flux defect extraction. RT successfully extracts features from PCB images and gain feature vector description, which can be used for classification.

The study shows the feasibility and capability of using MLP algorithmic models for PCB classification, among which the proposed approach attains the highest 
classification performance with less computational complexity. Based on the performance results, the Radon feature vector descriptors and MLP model classification are validated and proven to be a robust mechanism for the classification and recognition of flux defects in the dataset of PCB images.

The real-time AIS-PCB machine vision system is capable of sorting PCB flux defects based on texture with machine vision processing. The AIS-PCB system is developed based on various image-processing techniques using imaging software to classify the PCB flux defects as either accepted or rejected.

\section{Acknowledgement}

This research was supported by the Universiti Teknikal Malaysia Melaka (UTeM). The design, fabrication and software development were conducted by Control Easy Technology Sdn. Bhd (Control Easy).

\section{Funding Information}

This research was funded by $\mathrm{MyPhD}$ program, RMKe-10, Ministry Higher Education Malaysia Grant KPT(B)691115075391.

\section{Author's Contributions}

All authors equally contributed in this work.

\section{Ethics}

The article is original and contains unpublished. The corresponding author confirms that all the other authors have read the manuscript and approved it for publication.

\section{References}

Aouache, M., H. Aini and A.S. Salina, 2011. A new approach for noise reduction in spine radiograph images using a non-linear contrast adjustment scheme based adaptive factor. Scientific Res. Essays, 6: 4246-4258.

Bhardwaj, S.C., 2012. Machine vision algorithm for PCB parameters inspection. Proceedings of the National Conference on Future Aspects of Artificial Intelligence in Industrial Automation, (IIA' 12), IJCA.

Bradley, A.P., 1997. The use of the area under the ROC curve in the evaluation of machine learning algorithms. Patt. Recogn., 30: 1145-1159. DOI: $10.1016 / \mathrm{S} 0031-3203(96) 00142-2$

Brown, C.D. and H.T. Davis, 2006. Receiver operating characteristics curves and related decision measures: A tutorial. Chemometr. Intell. Laboratory Syst., 80: 24-38. DOI: 10.1016/j.chemolab.2005.05.004
Chauhan, A.P.S. and S.C. Bhardwaj, 2011. Detection of bare PCB defects by image subtraction method using machine vision. Proceedings of the World Congress on Engineering, Jul. 6-8, London, U.K.

Greenberg, A., G. Gutarts, A. Yaari, M. Barel and J. Nadivi, 2001. Method for printed circuit board inspection. Patent Application Publication.

Hagan, M.T., H.B. Demuth and M.H. Beale, 1996. Neural Network Design. 1st Edn., Pws, Boston.

Hilund, C., 2007. The radon transform. Aalborg University, Vision, Graphics and Interactive Systems.

Hussain, A., M.H. Sukairi, A. Mohamed and R. Mohamed, 2001. Automatic detection of power quality disturbances and identification of transient signals. Proceedings of the 6th International Symposium on Signal Processing and its Applications, Aug. 13-16, IEEE Xplore Press, Kuala Lumpur, pp: 462-465. DOI: $10.1109 /$ ISSPA.2001.950180

Kaushik, S. and J. Ashraf, 2012. Automatic PCB defect detection using image subtraction method. Int. J. Comput. Sci. Netw.

Leta, F.R., F.F. Feliciano, I.L. de Souza and E. Cataldo, 2005. Discussing accuracy in an automatic measurement system using computer vision techniques. Proceedings of the 18th International Congress of Mechanical Engineering (CME' 05).

Mar, N.S.S., C. Fookes and P.K. Yarlagadda, 2009. Design of automatic vision-based inspection system for solder joint segmentation. J. Achievements Mater. Manufactur. Eng., 34: 145-151.

Mashohor, S., J.R. Evans and T. Arslan, 2004. Genetic algorithm based Printed Circuit Board (PCB) inspection system. Proceedings of the IEEE International Symposium on Consumer Electronics, Sep. 1-3, IEEE Xplore Press, pp: 519-522.

DOI: 10.1109/ISCE.2004.1376000

Negnevitsky, M., 2005. Artificial Intelligence: A Guide to Intelligent Systems. 1st Edn., Addison-Wesley, New York, ISBN-10: 0321204662, pp: 415.

Purves, R.D., 1992. Optimum numerical integration methods for estimation of Area-Under-the-Curve (AUC) and Area-Under-the-Moment-Curve (AUMC). J. Pharmacokinet. Biopharmaceut., 20: 211-226. DOI: 10.1007/BF01062525

Rafiq, M.Y., G. Bugmann and D.J. Easterbrook, 2001. Neural network design for engineering applications. Comput. Struct., 79: 1541-1552. DOI: 10.1016/S0045-7949(01)00039-6

Singh, S. and M. Bharti, 2012. Image processing based automatic visual inspection system for PCBs. IOSR J. Eng., 2: 1451-1455. 
Sovierzoski, M.A., F.I. Argoud and F.M DeAzevedo, 2008. Evaluation of ANN classifiers during supervised training with roc analysis and cross validation. Proceedings of the International Conference on BioMedical Engineering and Informatics, May 27-30, IEEE Xplore Press, Sanya, pp: 274-278. DOI: 10.1109/BMEI.2008.251

Sundaraj, K., 2009. PCB inspection for missing or misaligned components using background subtraction. WSEAS Trans. Inform. Sci. Applic., 6: 778-787.

Teoh Ong, A., Z.B. Ibrahim and S. Ramli, 2013. Computer machine vision inspection on printed circuit boards flux defects. Am. J. Eng. Applied Sci., 6: 263-273. DOI: 10.3844/ajeassp.2013.263.273

Tilbury, J.B., W.J. Van Eetvelt, J.M. Garibaldi, J.S. Curnsw and E.C. Ifeachor, 2000. Receiver operating characteristic analysis for intelligent medical systems-a new approach for finding confidence intervals. IEEE Trans. Biomed. Eng., 47: 952-963. PMID: 10916267
Tsai, D.M. and Y.H. Tsai, 2002. Rotation-invariant pattern matching with color ring-projection. Patt. Recogn., 35: 131-141. DOI: 10.1016/S0031-3203(00)00180-1

Yager, R.R. and L.A. Zadeh, 1994. Fuzzy sets, neural networks and soft computing. John Wiley and Sons, Inc.

Zeng, Z., L. Ma and M. Suwa, 2011. Algorithm of locating PCB components based on colour distribution of solder joints. Int. J. Adv. Manufactur. Technol., 53: 601-614.

DOI: $10.1007 / \mathrm{s} 00170-010-2850-9$ 